\title{
Science to practice: translating automated brain MRI volumetry in Alzheimer's disease from research to routine diagnostic use in the work-up of dementia
}

\author{
Bharath Gopal Rathakrishnan ${ }^{1}$, P. Murali Doraiswamy ${ }^{2}$ and Jeffrey R. Petrella ${ }^{3}$ \\ 1 Duke University School of Medicine, Durham, NC, USA \\ ${ }^{2}$ Department of Psychiatry, Duke Institute for Brain Sciences, Duke University Medical Center, Durham, NC, USA \\ ${ }^{3}$ Department of Radiology, Duke University Medical Center, Durham, NC, USA \\ ${ }^{*}$ Correspondence: bg.rathakrishnan@gmail.com
}

Edited by:

José Julio Rodríguez Arellano, University of the Basque Country, Spain

Keywords: hippocampal volumetry, dementia, Alzheimer's disease, medial temporal lobe, medial temporal lobe atrophy, volumetrics

The medial temporal lobe (MTL) has long been identified as a key region affected by neurodegenerative diseases, a common source of cognitive decline in the elderly. The importance of the MTL with respect to cognitive decline is furthered by the hippocampus's role in memory formation (1). Impairment of declarative memory is one of the earliest symptoms of Alzheimer's disease (AD), the most common form of dementia. Early involvement of the MTL in $\mathrm{AD}$ is characterized by the presence of neurofibrillary tangles in the entorhinal cortex. As the disease progresses, neurofibrillary tangles continue to accumulate and involve the hippocampus, amygdala, and ultimately the neocortex (2). This gradual progression mirrors the worsening symptoms patients with $\mathrm{AD}$ experience. While initial concerns include memory deterioration, patients in later stages of $\mathrm{AD}$ can present with progressive aphasia (3), visuospatial impairments including agnosia (4), and apraxia (5).

Neuroimaging findings from Alzheimer's patients are consistent with the pathologic and clinical pattern, suggesting potential clinical diagnostic use. Structural magnetic resonance (MR) imaging analysis of patients with mild cognitive impairment (MCI) and $\mathrm{AD}$ is most notable for MTL atrophy, including significant volume reduction in the hippocampus, amygdala, parahippocampal gyrus, and entorhinal cortex (6). Structural MRI has identified the entorhinal cortex as the first region to display atrophy in AD. Atrophy in this region is followed by atrophy in the hippocampus, amygdala, and parahippocampal gyrus (7). The next region to display atrophy includes the posterior cingulate cortex; the atrophy then becomes generalized to the temporal neocortex and neocortical association areas (8). Thus, the evolution of neuroimaging findings on MRI closely mirrors the progression of neurodegenerative pathology, suggesting that volume assessment by MRI may be a useful clinical tool for early diagnosis and disease monitoring.

Qualitative visual assessment of temporal lobe atrophy on MRI has demonstrated a sensitivity of approximately $80 \%$ in distinguishing $\mathrm{AD}$ from normal aging, depression, and vascular dementia (9). A recent study revealed that qualitative visual assessment of MTL atrophy differentiated pathologically confirmed $\mathrm{AD}$ from vascular cognitive impairment and dementia with Lewy bodies with a sensitivity of $91 \%$ and specificity of $94 \%$ (10). Atrophy of the MTL is particularly important because it can be detected earlier than generalized whole brain atrophy in $\mathrm{AD}$. Unfortunately, there is significant variation in qualitative assessment of MTL atrophy among radiologists. One investigation of cognitively normal elderly individuals revealed a $70 \%$ interobserver agreement (kappa values of 0.59 and 0.62 ) in identifying the presence of MTL atrophy on coronal T1-weighted MRI scans (11). Another study included elderly patients with dementia and the interobserver agreement dropped to $49.7 \%$, with kappa values of 0.34 and 0.24 (12). Such poor inter-rater reliability is a major limitation for implementing qualitative MTL atrophy assessments in a clinical setting on individual patients.
This limitation has been addressed by quantitative volumetric analysis of MTL structures, which offers improved reliability and predictive accuracy. A comparison of qualitative ratings of MTL atrophy and hippocampal volumetry revealed that volumetry was more accurate in predicting cognitive decline to $\mathrm{AD}$ in MCI patients (100 vs. $78 \%$ positive predictive value, 100 vs. $87 \%$ negative predictive value) (13). A challenge of using any MTL atrophy assessment is that these structures often display volume loss in cognitively normal patients, much of which is attributed to the normal aging process. However, the effect of AD on MTL atrophy is much greater. Even patients with mild $\mathrm{AD}$ display significantly greater volume loss in MTL structures when compared to cognitively normal controls (14). The specific structure within the MTL that has demonstrated the most accuracy in predicting the presence of $\mathrm{AD}$ is the hippocampus. A semi-automated tracing-threshold technique for segmenting the hippocampus yielded a sensitivity of $82 \%$ and specificity of $80 \%$ in identifying patients with $\mathrm{AD}$ as compared to controls (14). Serial volume measurements tracking hippocampal change over time show even greater effects compared to baseline values alone. Rates of hippocampal atrophy were found to be considerably greater in Alzheimer's patients $\left(-3.98 \%,-150 \mathrm{~mm}^{3} /\right.$ year $)$ as compared to control patients $(-1.55 \%$, $-75 \mathrm{~mm}^{3} /$ year) (15). Additionally, hippocampal atrophy rates were found to correlate with patients' baseline cognitive statuses and also matched cognitive decline, with atrophy rates increasing from controls 

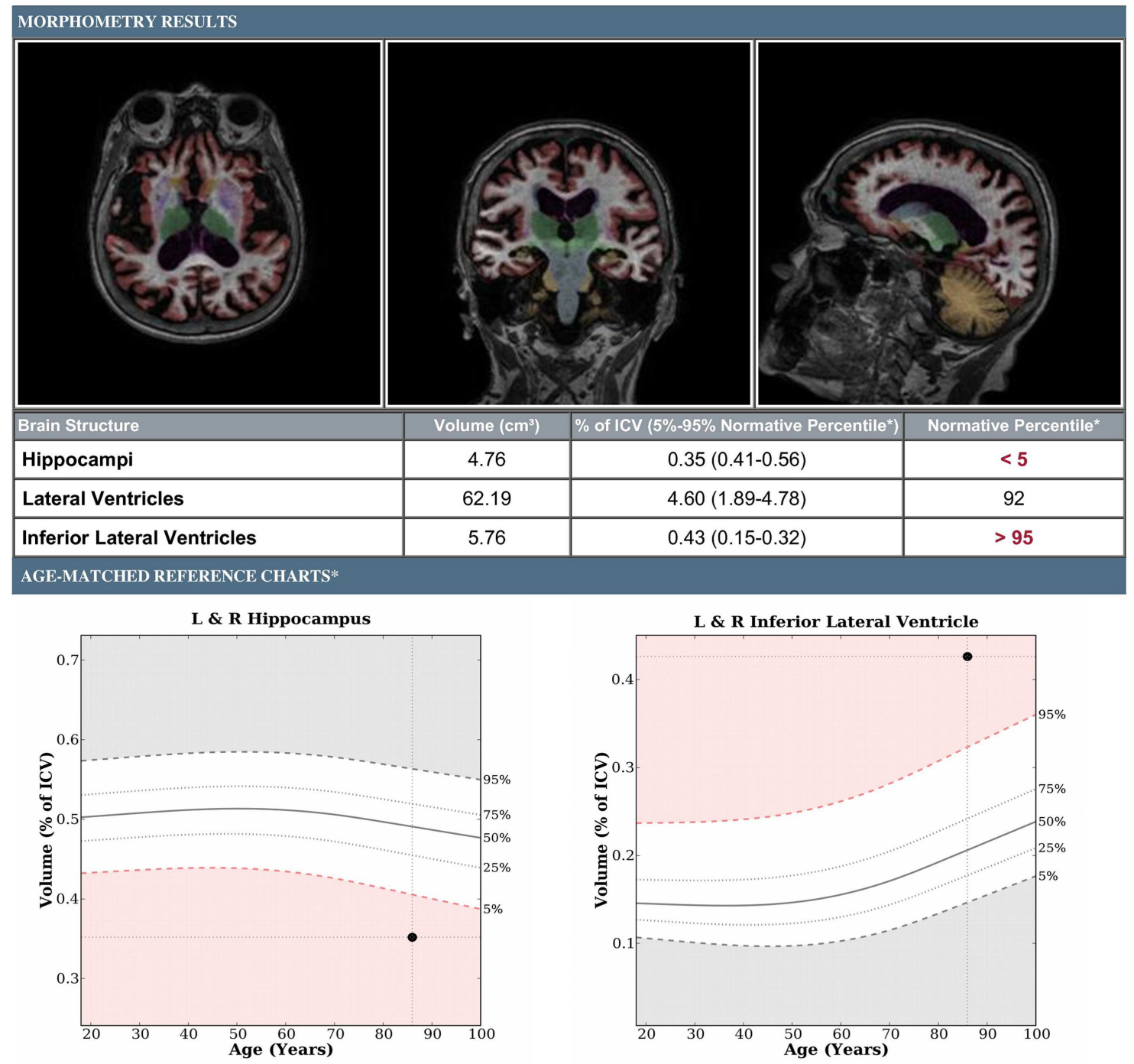

${ }^{*}$ Charts and normative values are provided for reference purposes only. The FDA has not approved their use for diagnostic purposes.

(C) CorTechs Labs, Inc. | www.cortechslabs.com

FIGURE 1 | Patient is an 85-year-old female with a 3-year history of memory loss with differential diagnosis of normal aging vs. mild Alzheimer's disease vs. vascular dementia. Figure demonstrates morphometry results displaying axial, coronal, and sagittal color-segmented images of the brain as well as volumetrics expressed in absolute and normalized units. Graphs demonstrate normalized volumes plotted against percentiles for age for the hippocampi and temporal horns. Results demonstrate hippocampal volume below the 5th percentile and temporal horns above the 95th percentile, supporting provisional diagnosis of mild Alzheimer's disease.
(1.73\% for stable, $2.81 \%$ for decliners) to MCI (2.55\% for stable, $3.69 \%$ for decliners) to $\operatorname{AD~}(3.5 \%)$ (16).

While studies have established this clear finding of hippocampal atrophy in $\mathrm{AD}$, hippocampal volumetry has not yet become a routine part of the diagnostic work-up for neurodegenerative diseases. Volumetric calculations required time-consuming, labor-intensive manual tracing over the borders of MTL structures over multiple slices. Summed regions of interest over the slices are used to count the number of voxels and ultimately produce a volume measurement. Despite the advantages over visual ratings, this method requires individual segmentation of structures within the MTL which may also vary considerably across different operators, depending upon their training and experience. Moreover, it is unclear how to account for the aging process and differences in head size in interpretation of such volume measurements in individual patients.

These concerns have been addressed through the development of software programs such as FreeSurfer, Individual Brain Atlases using statistical parametric mapping (IBASPM), and NeuroQuant that allow for completely automated, userindependent calculations of neural volumes. NeuroQuant, a commercial implementation of the FreeSurfer algorithm, also 
generates an age-related atrophy percentile, in addition to a general morphometry report (Figure 1). The age-related atrophy report includes the volume, percentage of intracranial volume, and normative percentiles for the hippocampus, lateral ventricles, and inferior lateral ventricles (temporal horns). The normative percentiles are based on control subjects from the multicenter $\mathrm{AD}$ Neuroimaging Initiative database (17). Implementations of these programs operate without user input and allow for rapid volumetric calculation and report generation immediately after a patient's MRI scan has been obtained, making them well-suited for incorporation into a busy clinical workflow.

Several of these software packages have been validated in studies comparing Alzheimer's patients to cognitively normal controls. Volumetric analysis was performed in both groups of patients using NeuroQuant vs. semi-manual segmentation as a gold standard. There was a high degree of agreement in the volumes generated (hippocampus, ICC $=0.93$, inferior lateral ventricle, $\mathrm{ICC}=0.92)$. Both methods demonstrated volume loss of the hippocampus and associated increased inferior lateral ventricle size in Alzheimer's patients as compared to controls (18). FreeSurfer has also been incorporated in assessment of temporal lobe structures in patients with $\mathrm{AD}$ and semantic dementia. One investigation revealed that FreeSurfer correlated highly with manual volumetric delineation, particularly in the ventricles $(r=0.99)$, right medial-inferior temporal gyrus $(r=0.84)$, and left hippocampus $(r=0.76)$ (19).

Further investigations are needed to elucidate the clinical role and utility of automated hippocampal volumetry. While studies have demonstrated the sensitivity of hippocampal and MTL atrophy for conditions such as MCI and AD, hippocampal atrophy can occur in a number of other settings. Hippocampal atrophy has been documented in numerous conditions ranging from Cushing's syndrome, major depression, posttraumatic stress disorder (20), Type 2 Diabetes (21), traumatic brain injury, and concussions (22). Research has also implicated several genotypes associated with hippocampal volumes. Val66Met polymorphisms of the BDNF gene have been linked to worsened episodic memory
(23) and reduced hippocampal volumes (24), suggesting BDNF's role in vulnerability to neural changes associated with aging (25). The APOE $\varepsilon 4$ allele has also demonstrated an association with increased rate (26) and amount (27) of hippocampal atrophy. Decreased hippocampal volumes in patients with depression were linked to 44-base pair insertions in the promoter region of the serotonin transporter (5HTTLPR) (28). Therefore, additional work is needed to establish the specificity of hippocampal atrophy in a general practice setting, including patients with multiple, sometimes coexisting, conditions, and genotypes.

In addition, while we believe hippocampal volumetry can be incorporated into neuroimaging evaluation of patients with potential neurodegenerative diseases, significant work still needs to be done to establish standardized volumetry thresholds for atrophy rates and age-adjusted volumes that will be useful in practice for different clinical contexts, such as differential diagnosis, early identification/prediction, and disease monitoring. A clear delineation of hippocampal boundaries is required along with a standardized, accessible database, and uniform methods of reporting hippocampal volumes (29). Hippocampal volumetry still awaits U.S. FDA approval for specific clinical contexts of use because of the current lack of standardized volumetry norms. Nevertheless, the European Medicines Agency has approved the use of this technique for sample enrichment in clinical trials (30). Though full incorporation in routine practice awaits such norms, complete hierarchical validation (31) may unnecessarily delay implementation; therefore, alternative approaches should be considered for concurrent assessment and implementation of this technology (32). Hippocampal volumetrics may currently be helpful as an aid in differential diagnosis of dementia and MCI in specific cases, particularly when values fall in the normal range. Hippocampal volumetry may be particularly important in identifying patients in the pre-clinical stages of $\mathrm{AD}$ and also in predicting worsening of MCI patients. Indeed, new diagnostic criteria have been proposed for both $\mathrm{AD}$ and MCI due to $\mathrm{AD}$ which incorporate MRI volumetry as a neuronal loss marker (33). Thus, the time is now for hippocampal volumetry to become a component of the neuroimaging evaluation of patients with suspected neurodegenerative disease.

\section{ACKNOWLEDGMENTS}

P. Murali Doraiswamy has received research grants and served as an adviser to several healthcare and pharmaceutical companies. He owns stock in Sonexa, Clarimedix, and Maxwell Health whose products are not discussed here. Jeffrey R. Petrella has received research grants and served as an adviser to several healthcare and pharmaceutical companies including Quintiles (speakers bureau), Janssen (advisory board), and Piramal (advisory board), whose products are not discussed here.

\section{REFERENCES}

1. Squire LR. Memory and the hippocampus: a synthesis from findings with rats, monkeys, and humans. Psychol Rev (1992) 99(2):195-231. doi: 10.1037/0033-295X.99.3.582

2. Braak E, Griffing K, Arai K, Bohl J, Bratzke H, Braak H. Neuropathology of Alzheimer's disease: what is new since A. Alzheimer? Eur Arch Psychiatry Clin Neurosci (1999) 249(3):14-22. doi:10.1007/ PL00014168

3. Galton CJ, Patterson K, Xuereb JH, Hodges JR. Atypical and typical presentations of Alzheimer's disease: a clinical, neuropsychological, neuroimaging and pathological study of 13 cases. Brain (2000) 123(3):484-98. doi:10.1093/brain/123.3.484

4. Mendez MF, Mendez MA, Martin R, Smyth KA, Whitehouse PJ. Complex visual disturbances in Alzheimer's disease. Neurology (1990) 40(3 Pt 1):439-43. doi:10.1212/WNL.40.3_Part_1.439

5. Parakh R, Roy E, Koo E, Black S. Pantomine and imitation limb gestures in relation to the severity of Alzheimer's disease. Brain Cogn (2004) 55(2):272-4. doi:10.1016/j.bandc.2004.02.049

6. Petrella JR, Coleman RE, Doraiswamy PM. Neuroimaging and early diagnosis of Alzheimer disease: a look to the future. Radiology (2003) 226(2):315-36. doi:10.1148/radiol.2262011600

7. Killiany RJ, Hyman BT, Gomez-Isla T, Moss MB, Kikinis R, Jolesz F, et al. MRI measures of entorhinal cortex vs hippocampus in preclinical AD. Neurology (2002) 58(8):1188-96. doi:10.1212/WNL. 58.8.1188

8. Scahill RI, Schott JM, Stevens JM, Rossor MN, Fox NC. Mapping the evolution of regional atrophy in Alzheimer's disease: unbiased analysis of fluidregistered serial MRI. Proc Natl Acad Sci U S A (2002) 99(7):4703-7. doi:10.1073/pnas.052587399

9. O’Brien JT, Desmond P, Ames D, Schweitzer I, Chiu E, Tress B. Temporal lobe magnetic resonance imaging can differentiate Alzheimer's disease from normal aging, depression, vascular dementia, and other causes of cognitive impairment. Psychol Med (1997) 27(6):1267-75. doi:10. 1017/S0033291797005606

10. Burton EJ, Barber R, Mukaetova-Ladinska EB, Robson J, Perry RH, Jaros E, et al. Medial temporal 
lobe atrophy on MRI differentiates Alzheimer's disease from dementia with Lewy bodies and vascular cognitive impairment: a prospective study with pathological verification of diagnosis. Brain (2009) 132(1):195-203. doi:10.1093/brain/ awn 298

11. Scheltens P, Launer LJ, Barkhof F, Weinstein $\mathrm{HC}$, van Gool WA. Visual assessment of medial temporal lobe atrophy on magnetic resonance imaging: interobserver reliability. J Neurol (1995) 242(9):557-60. doi:10.1007/BF00868807

12. Scheltens P, Pasquier SP, Weerts JG, Barkhof F, Leys D. Qualitative assessment of cerebral atrophy on MRI: inter- and intra-observer reproducibility in dementia and normal aging. Eur Neurol (1997) 37(2):95-9. doi:10.1159/000117417

13. Visser PJ, Verhey FR, Hofman PA, Scheltens P, Jolles J. Medial temporal lobe atrophy predicts Alzheimer's disease in patients with minor cognitive impairment. J Neurol Neurosurg Psychiatry (2002) 72(4):491-7. doi:10.1136/jnnp.72.4.491

14. Jack CR, Petersen RC, Xu YC, Waring SC, O’Brien PC, Tangalos EG, et al. Medial temporal atrophy on MRI in normal aging and very mild Alzheimer's disease. J Neurol (1997) 49(3):786-94. doi:10.1212/WNL.49.3.786

15. Jack CR, Petersen RC, Xu Y, O’Brien PC, Smith GE, Ivnik RJ, et al. Rate of medial temporal lobe atrophy in typical aging and Alzheimer's disease. $J$ Neurol (1998) 51(4):993-9. doi:10.1212/WNL.51. 4.993

16. Jack CR Jr, Petersen RC, Xu Y, O’Brien PC, Smith GE, Ivnik RJ, et al. Rates of hippocampal atrophy correlate with change in clinical status in aging and Alzheimer's disease. J Neurol (2000) 55(4):484-9. doi:10.1212/WNL.55.4.484

17. NeuroQuant. Cortechs labs. (2009). Available from: http://www.cortechs.net/products/ neuroquant.php

18. Brewer JB, Magda S, Airriess C, Smith ME. Fully automated quantification of regional brain volumes for improved detection of focal atrophy in Alzheimer's disease. AJNR Am J Neuroradiol (2009) 30(3):578-80. doi:10.3174/ajnr.A1402

19. Lehmann M, Douiri A, Kim LG, Modat M, Chan D, Ourselin S, et al. Atrophy patterns in Alzheimer's disease and semantic dementia: a comparison of FreeSurfer and manual volumetric measurements. Neuroimage (2010) 49(3):2264-74. doi:10.1016/j. neuroimage.2009.10.056
20. Sapolsky RM. Glucocorticoids and hippocampal atrophy in neuropsychiatric disorders. Arch Gen Psychiatry (2000) 57(10):925-35. doi:10.1001/ archpsyc.57.10.925

21. den Heijer TD, Vermeer SE, van Dijk EJ, Prins ND, Koudstaal PJ, Hofman A, et al. Type 2 diabetes and atrophy of medial temporal lobe structures on brain MRI. Diabetologia (2003) 46(12):1604-10. doi:10.1007/s00125-003-1235-0

22. Himanen L, Portin R, Isoniemi $\mathrm{H}$, Helenius $\mathrm{H}$, Kurki T, Tenovu O. Cognitive functions in relation to MRI findings 30 years after traumatic brain injury. Brain Inj (2005) 19(2):93-100. doi:10.1080/ 02699050410001720031

23. Seidah NG, Benjannet S, Pareek S, Chrétien M, Murphy RA. Cellular processing of the neurotrophin precursors of NT3 and BDNF by the mammalian proprotein convertases. FEBS Lett (1996) 379(3):247-50. doi:10.1016/00145793(95)01520-5

24. Pezawas L, Verchinski BA, Mattay VS, Callicott JH, Kolachana BS, Straub RE, et al. The brainderived neurotrophic factor val66met polymorphism and variation in human cortical morphology. J Neurosci (2004) 24(45):10099-102. doi:10. 1523/JNEUROSCI.2680-04.2004

25. Petrella JR, Mattay VS, Doraiswamy PM. Imaging genetics of brain longevity and mental wellness: the next Frontier? Radiology (2008) 246(1):20-32. doi:10.1148/radiol.2461061994

26. Moffat SD, Szekely CA, Zonderman AB, Kabani NJ, Resnick SM. Longitudinal change in hippocampal volume as a function of apolipoprotein E genotype. J Neurol (2000) 55(1):134-6. doi:10.1212/ WNL.55.1.134

27. Lehtovirta M, Laakso MP, Soininen H, Helisalmi S, Mannermaa A, Helkala EL, et al. Volumes of hippocampus, amygdala, and frontal lobe in Alzheimer patients with different apolipoprotein E genotypes. Neuroscience (1995) 67(1):65-72. doi:10.1016/0306-4522(95)00014-A

28. Taylor WD, Steffens DC, Payne ME, MacFall JR, Marchuk DA, Svenson IK, et al. Influence of serotonin transporter promoter region polymorphisms on hippocampal volumes in late-life depression. Arch Gen Psychiatry (2005) 62(5):537-44. doi:10.1001/archpsyc.62.5. 537

29. Jack CR Jr, Barkhof F, Bernstein MA, Cantillon M, Cole PE, Decarli C, et al. Steps to standardization and validation of hippocampal volumetry as a biomarker in clinical trials and diagnostic criteria for Alzheimer's disease. Alzheimers Dement (2011) 7(4):474-85. doi:10.1016/j.jalz. 2011.04.007

30. Trelles FDA, Pani L, Jonsson B, European Medicines Agency, C-Path CAMD Biomarker Working Group. Qualification opinion of low hippocampal volume (atrophy) by MRI for use in regulatory clinical trials - in pre-dementia stage of Alzheimer's disease. (2011). Available from: http://www.ema.europa.eu/docs/en_GB/ document_library/Regulatory_and_procedural_ guideline/2011/10/WC500116264.pdf

31. Fryback DG, Thornbury JR. The efficacy of diagnostic imaging. Med Decis Making (1991) 11(2):88-94. doi:10.1177/0272989X9101100203

32. Hunink MG, Krestin GP. Study design for concurrent development, assessment, and implementation of new diagnostic imaging technology. Radiology (2002) 222(3):604-14. doi:10.1148/radiol. 2223010335

33. Jack CR, Albert MS, Knopman DS, McKhann GM, Sperling RA, Carrillo MC, et al. Introduction to recommendations from the National Institute on Aging-Alzheimer's Association workgroups on diagnostic guidelines for Alzheimer's disease. Alzheimers Dement (2011) 7(3):257-62. doi:10.1016/j.jalz.2011.03.004

Received: 07 October 2013; accepted: 23 December 2013; published online: 09 January 2014.

Citation: Rathakrishnan BG, Doraiswamy PM and Petrella JR (2014) Science to practice: translating automated brain MRI volumetry in Alzheimer's disease from research to routine diagnostic use in the work-up of dementia. Front. Neurol. 4:216. doi: 10.3389/fneur.2013.00216

This article was submitted to Neurodegeneration, a section of the journal Frontiers in Neurology.

Copyright (c) 2014 Rathakrishnan, Doraiswamy and Petrella. This is an open-access article distributed under the terms of the Creative Commons Attribution License (CC BY). The use, distribution or reproduction in other forums is permitted, provided the original author(s) or licensor are credited and that the original publication in this journal is cited, in accordance with accepted academic practice. No use, distribution or reproduction is permitted which does not comply with these terms. 